\title{
Efficacy Evaluation of Early, Low-Dose, Short-Term Corticosteroids in Adults Hospitalized with Non- Severe COVID-19 Pneumonia: A Retrospective Cohort Study
}

\author{
Qiang Li · Weixia Li · Yinpeng Jin · Wei Xu • Chenlu Huang · \\ $\mathrm{Li} \mathrm{Li} \cdot$ Yuxian Huang $\cdot$ Qingchun Fu $\cdot$ Liang Chen
}

Received: July 7, 2020 / Published online: September 2, 2020

(C) The Author(s) 2020

\section{ABSTRACT}

Objectives: This study aimed to observe the efficacy of corticosteroids in non-severe COVID19 pneumonia.

Methods: A retrospective study based on propensity score matching was designed to explore the effects of corticosteroids. Primary outcomes included the rate of patients who developed severe disease and mortality. Secondary outcomes included duration of fever, virus clearance time, length of hospital stay, and the use of antibiotics.

Digital Features To view digital features for this article go to https://doi.org/10.6084/m9.figshare.12834902.

Qiang Li, Weixia Li, Yinpeng Jin, Wei Xu and Chenlu Huang contributed equally.

Q. Li · Y. Jin · W. Xu · C. Huang · L. Li · Y. Huang ·

Q. Fu $(\square) \cdot$ L. Chen $(\square)$

Department of Liver Disease, Shanghai Public

Health Clinical Center, Fudan University, Shanghai, China

e-mail: fuqingchun@shphc.org.cnL. Chen

e-mail: chenliang@shphc.org.cn

W. Li

Department of Infectious Disease, Shanghai Public Health Clinical Center, Fudan University, Shanghai, China
Results: A total of 475 patients with non-severe COVID-19 pneumonia were enrolled, 55 patients received early, low-dose, and shortterm corticosteroids therapy, 420 patients received non-corticosteroids therapy. Compared to the non-corticosteroids group, there was a prolonged duration of fever (median 5 vs 3 days, $p<0.001$ ), virus clearance time (median 18 vs 11 days, $p<0.001$ ), and length of hospital stay (median 23 vs 15 days, $p<0.001$ ) in the corticosteroids group. The percentages of antibiotics therapy $(89.1 \%$ vs $23.6 \%, p<0.001)$, use of at least two antibiotics (38.2\% vs $12.7 \%$, $p=0.002)$, and antifungal therapy $(7.3 \%$ vs 0 , $p=0.042$ ) were higher in the corticosteroids group than those in the non-corticosteroids group. Compared to the non-corticosteroids group, more patients developed severe disease $(12.7 \%$ vs $1.8 \%, p=0.028)$ in the corticosteroids group. There was no significant difference between the two groups in mortality $(1.8 \%$ vs 0 , $p=0.315$ ).

Conclusion: In adult patients with non-severe COVID-19 pneumonia, early, low-dose, and short-term corticosteroids therapy was associated with worse clinical outcomes.

Keywords: 2019 novel coronavirus disease; Corticosteroids; Efficacy evaluation; Nonsevere COVID-19 infections; Severe acute respiratory syndrome coronavirus 2 


\section{Key Summary Points}

At present, there is an absence of any proven effective antiviral therapy. This study aimed to observe the efficacy of corticosteroids in non-severe COVID-19 pneumonia.

The use of corticosteroids increased the risk of COVID-19 progression to severe disease, increased the use of antibiotics, and prolonged duration of fever, virus clearance time, and length of hospital stay in non-severe COVID-19 pneumonia.

In hospitalized adult non-severe COVID19 pneumonia, early, low-dose, and shortterm corticosteroids were associated with worse clinical outcomes. Our results did not support the use of corticosteroids in non-severe COVID-19 pneumonia.

\section{DIGITAL FEATURES}

This article is published with digital features to facilitate understanding of the article. You can access the digital features on the article's associated Figshare page. To view digital features for this article go to https://doi.org/10.6084/m9. figshare.12834902.

\section{INTRODUCTION}

Since December 2019, the severe acute respiratory syndrome coronavirus 2 (SARS-CoV-2) has caused an outbreak of 2019 novel coronavirus disease (COVID-19) [1]. To date, SARS-CoV-2 has spread to over 200 countries and areas worldwide, and the number of confirmed cases and death cases has been quickly growing. As of July 4, 2020, there have been 10,922,324 confirmed cases and 523,011 deaths globally [2]. The disease spectrum of COVID-19 ranges from mild self-limiting disease to severe disease necessitating hospitalization and intensive care unit (ICU) [3]. At present, there is an absence of any proven effective antiviral therapy.

The main pathological changes of SARSCoV-2 infection are characterized by ongoing lung inflammation, exudative pulmonary edema, which may be responsive to corticosteroids therapy [4]. Corticosteroids may diminish the inflammatory response, a major factor for lung damage and acute respiratory distress syndrome (ARDS) in SARS-CoV-2 infection. Use of corticosteroids-based therapy to reduce inflammatory-induced lung injury has been described for patients with severe COVID-19 $[1,5]$, similar to the use of corticosteroids to treat severe acute respiratory syndrome (SARS) in 2003 [6]. Russell et al. [7] reported that there appeared to be some evidence that corticosteroids may be beneficial if utilized in the early acute phase of SARS-CoV-2 infection; however, conflicting evidence from the World Health Organization (WHO) surrounding corticosteroids use in SARS-CoV-2 infection means this evidence is not conclusive. Han et al. [8] reported that the long-term use of corticosteroids might cause atypical infections, a long incubation period, and extra transmission of COVID-19. Tang et al. [9] call for caution in the use of corticosteroids for COVID-19 and do not recommend corticosteroids as a routine treatment.

For non-severe COVID-19 pneumonia, should corticosteroids be forbidden? What would be the advantages and disadvantages of corticosteroids therapy in non-severe COVID-19 pneumonia? In the early stages of the outbreak of COVID-19, early, low-dose, and short-term corticosteroids were used in partial non-severe cases with COVID-19 pneumonia in our hospital. In this retrospective study, we compared the clinical outcomes of patients with non-severe COVID-19 pneumonia treated with and without corticosteroids.

\section{METHODS}

\section{Patients}

A total of 664 consecutive patients with COVID19 hospitalized at the isolation ward of 


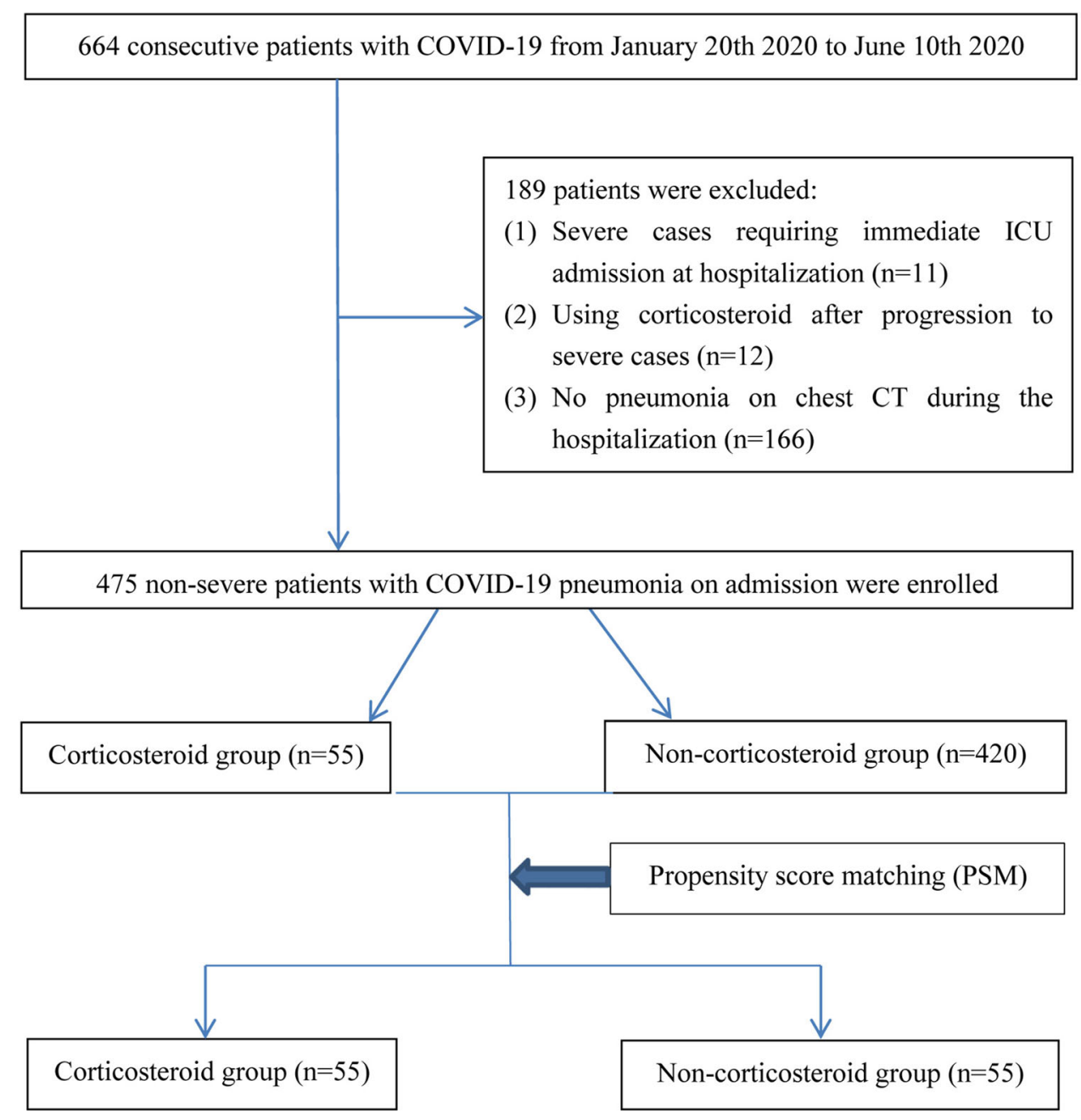

Fig. 1 Flow diagram of the study population. A total of 664 consecutive patients with COVID-19 were retrospectively analyzed. Finally, 475 patients with non-severe COVID-19 pneumonia were enrolled; among them, 55 patients received methylprednisolone therapy (corticosteroids group) and 420 patients received the standard

Shanghai Public Health Clinical Center from January 20, 2020 to June 10, 2020 were retrospectively analyzed. Shanghai Public Health Clinical Center is a tertiary teaching hospital in Shanghai, China, and is designated by the Chinese government for the treatment of adult patients with COVID-19. Inclusion criteria: (1) patients with confirmed COVID-19; (2) pneumonia on chest CT scan; (3) age 16 years or older. Exclusion criteria: (1) severe cases requiring immediate ICU admission at therapy (non-corticosteroids group). As significant differences existed in baseline characteristics between the corticosteroids group and non-corticosteroids group, 55 pairs were generated according to propensity score matching

hospitalization $(n=11)$; (2) using corticosteroids after progression to severe disease $(n=12)$; (3) no pneumonia on chest CT scan during the hospitalization $(n=166)$. Finally, 475 patients with non-severe COVID-19 pneumonia were enrolled. The flow chart is shown in Fig. 1. The clinical outcomes were monitored up to July 6, 2020, and all patients had been discharged or died during the hospitalization.

The ethics committee of Shanghai Public Health Clinical Center approved the study 
protocol and experiments, including any relevant details (YJ-2020-S095-01). Although this is a retrospective study, at admission, all patients provided verbal consent that their clinical data might be used for medical study. When we performed the study, all personal information of patients was de-identified to protect privacy. This study was performed in accordance with the declaration of Helsinki.

\section{Diagnostic Criteria}

In this study, all patients with COVID-19 were diagnosed according to the positive results of SARS-COV-2 nucleotides following the WHO guideline [10]. The nasopharyngeal or throat swab specimens were collected and the SARSCOV-2 nucleotides were tested using real-time polymerase chain reaction (RT-PCR) methods in the Chinese Center for Disease Prevention and Control. According to the novel coronavirus pneumonia prevention and control program published by the National Health Committee of China, non-severe COVID-19 pneumonia was defined as cases with pneumonia on radiological images, but did not meet any of the following [11]: (1) respiratory distress, respiratory rates $\geq 30 / \mathrm{min}$; (2) pulse oxygen saturation $\leq 93 \%$ in the resting state; (3) oxygenation index $\leq 300 \mathrm{mmHg}$; (4) require mechanical ventilation; (5) shock; (6) combined with other organ failures and needed treatment in the ICU.

\section{Outcomes and Definitions}

In this study, primary outcomes included the rate of patients who developed severe disease and mortality. Secondary outcomes included duration of fever, virus clearance time, length of hospital stay, and the use of antibiotics. The duration of fever was defined as the time from the onset of fever to the date of sustaining normal temperature. The virus clearance time was defined as the time from illness onset to two consecutive negative tests for SARS-CoV-2 with at least 24-h intervals.

\section{Data Collection}

The demographic characteristics, comorbidities, vital signs, chest CT findings, treatments (duration and dosage), virus clearance times, and length of hospital stay were extracted from electronic medical records. For patients in this study, the vital signs were monitored daily; laboratory tests were examined every 3 days; and chest CT scans were performed every 3-7 days. The treatments were collected including corticosteroids, antiviral drugs, and antibiotics, which were used for patients as appropriate.

\section{Statistical Analysis}

The normality test was performed for continuous variables using the Kolmogorov-Smirnov test. Normally distributed variables, non-normally distributed continuous variables, and categorical variables are reported as means and standard deviations, medians and interquartile ranges (IQR), and counts and percentage, respectively. $T$ tests, Mann-Whitney tests, and chi-square tests were applied to normally distributed variables, non-normally distributed continuous variables, and categorical variables, respectively. Propensity score matching (PSM) is a powerful tool for comparing groups with similar observed characteristics without specifying the relationship between confounders and outcomes [12]. We adjusted for differences in baseline characteristics of patients between the corticosteroids group and non-corticosteroids group using PSM. Propensity scores for all patients were estimated according to some covariates that might have affected patient assignment to a corticosteroids or non-corticosteroids group, as well as primary clinical outcomes. The effect of corticosteroids treatment on clinical outcomes was analyzed by multivariate logistic regression with adjustment for variables associated with primary clinical outcomes (developed severe disease or died) [13]. A one-to-one matched analysis using nearest-neighbor matching was performed on the basis of the estimated propensity scores of each patient and the caliper was set as 0.25 [14]. All statistical analyses were conducted with SPSS 
Table 1 Baseline characteristics of patients with or without corticosteroids therapy

\begin{tabular}{|c|c|c|c|c|}
\hline & All & Corticosteroids & Non-corticosteroids & $p$ value \\
\hline Number of patients & 475 & 55 & 420 & \\
\hline Age (years) & $42(32-58)$ & $59(46-68)$ & $40(31-55)$ & $<0.001$ \\
\hline Male, $n(\%)$ & $258(54.3 \%)$ & $33(60 \%)$ & $225(53.6 \%)$ & 0.368 \\
\hline Female, $n(\%)$ & $217(45.7 \%)$ & $22(40 \%)$ & $195(46.4 \%)$ & 0.368 \\
\hline Propensity score & - & $0.12(0.10-0.15)$ & $0.05(0.03-0.08)$ & $<0.001$ \\
\hline \multicolumn{5}{|l|}{ Vital signs } \\
\hline Temperature $\left({ }^{\circ} \mathrm{C}\right)$ & $37.0(36.8-37.2)$ & $37.7(37.2-38.2)$ & $36.9(36.7-37.2)$ & $<0.001$ \\
\hline Respiratory rates $(/ \mathrm{min})$ & $21(19-24)$ & $25(22-27)$ & $20(17-23)$ & $<0.001$ \\
\hline Heart rates $(/ \mathrm{min})$ & $75(67-82)$ & $88(79-94)$ & $73(65-82)$ & $<0.001$ \\
\hline $\mathrm{SBP}(\mathrm{mmHg})$ & $112(94-130)$ & $125(110-142)$ & $110(92-128)$ & $<0.001$ \\
\hline Oxygen saturation (\%) & 97 (95-98) & $95(94-97)$ & 97 (96-98) & $<0.001$ \\
\hline $\mathrm{FiO} 2(\%)$ & $33(33-33)$ & $41(33-41)$ & $33(33-33)$ & $<0.001$ \\
\hline Comorbidities, $n(\%)$ & $123(25.9 \%)$ & $23(41.8 \%)$ & $100(23.8 \%)$ & 0.004 \\
\hline Hypertension & $82(17.3 \%)$ & $19(34.5 \%)$ & $63(15 \%)$ & $<0.001$ \\
\hline Diabetes & $37(7.8 \%)$ & $6(10.9 \%)$ & $31(7.4 \%)$ & 0.359 \\
\hline $\mathrm{CHD}$ & $14(2.9 \%)$ & $3(5.5 \%)$ & $11(2.6 \%)$ & 0.242 \\
\hline CLD & $9(1.9 \%)$ & $1(1.8 \%)$ & $8(1.9 \%)$ & 0.965 \\
\hline $\mathrm{CPD}$ & $7(1.5 \%)$ & $1(1.8 \%)$ & $6(1.4 \%)$ & 0.822 \\
\hline $\mathrm{CKD}$ & $2(0.4 \%)$ & 0 & $2(0.5 \%)$ & 0.608 \\
\hline Malignancy & $3(0.6 \%)$ & 0 & $3(0.7 \%)$ & 0.530 \\
\hline \multicolumn{5}{|c|}{ Laboratory parameters at admission } \\
\hline $\operatorname{WBC}\left(10^{9} / \mathrm{L}\right)$ & $5.0(4.1-6.2)$ & $4.4(3.6-5.8)$ & $5.1(4.1-6.2)$ & 0.015 \\
\hline Lymphocyte $\left(10^{9} / \mathrm{L}\right)$ & $1.3(0.9-1.7)$ & $0.9(0.7-1.1)$ & $1.3(1.0-1.7)$ & $<0.001$ \\
\hline $\mathrm{CRP}(\mathrm{mg} / \mathrm{L})$ & $3.7(0.5-15.5)$ & $25.9(8.7-46.0)$ & $2.6(0.5-10.9)$ & $<0.001$ \\
\hline $\mathrm{LDH}(\mathrm{U} / \mathrm{L})$ & $213(188-255)$ & $285(215-357)$ & $209(185-243)$ & $<0.001$ \\
\hline $\mathrm{D}$-dimer $(\mathrm{ng} / \mathrm{mL})$ & $0.4(0.2-0.6)$ & $0.5(0.3-0.9)$ & $0.4(0.2-0.5)$ & 0.847 \\
\hline \multicolumn{5}{|l|}{ Antiviral therapy } \\
\hline Thymosin- $\alpha$ & $160(33.7 \%)$ & $46(83.6 \%)$ & $114(27.1 \%)$ & $<0.001$ \\
\hline Hydroxychloroquine & $153(32.2 \%)$ & $3(5.5 \%)$ & $150(35.7 \%)$ & $<0.001$ \\
\hline Arbidol & $135(28.4 \%)$ & $35(63.6 \%)$ & $100(23.8 \%)$ & $<0.001$ \\
\hline Lopinavir/ritonavir & $110(23.2 \%)$ & $31(56.4 \%)$ & $79(18.8 \%)$ & $<0.001$ \\
\hline
\end{tabular}


Table 1 continued

\begin{tabular}{lllll}
\hline & All & Corticosteroids & Non-corticosteroids & $\boldsymbol{p}$ value \\
\hline Emtricitabine/tenofovir & $12(2.5 \%)$ & 0 & $12(2.9 \%)$ & 0.204 \\
\hline
\end{tabular}

$p$ values indicate differences between the corticosteroids group and non-corticosteroids group

$S B P$ systemic blood pressure, $F i O 2$ fraction of inspired $\mathrm{O}_{2}, C H D$ chronic heart disease, $C L D$ chronic liver disease, $C P D$ chronic pulmonary disease, $C K D$ chronic kidney disease, $W B C$ white blood cell, $C R P$ C-reactive protein, $L D H$ lactate dehydrogenase

software version 15.0 (SPSS Inc. USA), and statistical significance set at two-sided $p<0.05$.

\section{RESULTS}

\section{Baseline Characteristics of Patients with or Without Corticosteroids Therapy}

Baseline characteristics of patients with or without corticosteroids therapy are shown in Table 1 . The median age was 42 years (IQR $32-58$ years); 258 patients were male $(54.3 \%)$, and 123 patients had comorbidities (28.9\%). The median white blood cell (WBC) count, lymphocyte count, C-reactive protein (CRP), lactate dehydrogenase (LDH), and D-dimer were $5.0 \times 10^{9} / \mathrm{L} \quad(\mathrm{IQR} \quad 4.1-6.2), 1.3 \times 10^{9} / \mathrm{L} \quad(\mathrm{IQR}$ 0.9-1.7), $3.7 \mathrm{mg} / \mathrm{L}$ (IQR 0.5-15.5), $213 \mathrm{U} / \mathrm{L}$ (IQR 188-255), and $0.4 \mathrm{ng} / \mathrm{mL}$ (IQR 0.2-0.6), respectively. Most patients received antiviral agents including thymosin- $\alpha$ (160, 33.7\%), hydroxychloroquine (153, 32.2\%), Arbidol (135, 28.4\%), lopinavir/ritonavir $(110,23.2 \%)$, and emtricitabine/tenofovir $(12,2.5 \%)$.

Patients who had a higher median temperature $\left(37.7^{\circ} \mathrm{C}\right.$ [IQR $37.2-38.2^{\circ} \mathrm{C}$ ] vs $36.9^{\circ} \mathrm{C}$ [IQR $\left.\left.36.7-37.2^{\circ} \mathrm{C}\right] ; p<0.001\right)$, respiratory rates $(25$ times/min [IQR 22-27 times/min] vs 20 times/ min [IQR 17-23 times/min]; $p<0.001$ ), heart rates ( 88 beats/min [IQR $79-94$ beats/min] vs 73 beats/min [IQR $65-82$ beats/min]; $p<0.001)$, systemic blood pressure $(125 \mathrm{mmHg}$ [IQR $110-142 \mathrm{mmHg}$ ] vs $110 \mathrm{mmHg}$ [IQR 92-$128 \mathrm{mmHg}$; $p<0.001), \quad \mathrm{FiO} 2 \quad(41 \%$ [IQR $33-41 \%$ ] vs 33\% [IQR 33-33\%]; $p<0.001$ ), but lower median oxygen saturation (95\% [IQR 94-97\%] vs 97\% [IQR 96-98\%]; $p<0.001$ ) were more likely to be treated with corticosteroids (Table 1).

As shown in Table 1, patients who had higher age (59 vs 40 years, $p<0.001$ ), more common comorbidities $(41.8 \%$ vs $23.8 \%$, $p=0.004)$, higher CRP $(25.9$ vs $2.6 \mathrm{mg} / \mathrm{L}$, $p<0.001)$, LDH (285 vs $209 \mathrm{U} / \mathrm{L}, p<0.001)$, but lower WBC $\left(4.4\right.$ vs $\left.5.1 \times 10^{9} / \mathrm{L}, p=0.015\right)$ and lymphocyte counts $\left(0.9\right.$ vs $1.3 \times 10^{9} / \mathrm{L}$, $p<0.001$ ), were more likely to be treated with corticosteroids. Moreover, thymosin- $\alpha$ (83.6\% vs $27.1 \%, p<0.001)$, Arbidol $(63.6 \%$ vs $23.8 \%$, $p<0.001)$, and lopinavir/ritonavir $(56.4 \%$ vs $18.8 \%, p<0.001$ ) were more frequently used in the corticosteroids group, while hydroxychloroquine $(5.5 \%$ vs $35.7 \%, p<0.001)$ was less common in the corticosteroids group.

\section{Details of Administration of Corticosteroids}

In this study, there were 55 patients who received corticosteroids therapy, and 420 patients who received standard therapy (noncorticosteroid therapy). Prednisone [methylprednisolone-equivalent dose, $20 \mathrm{mg} /$ day] was administered orally to 5 patients for 3 days, whereas methylprednisolone was administered intravenously to 50 patients. The dose and duration of intravenous methylprednisolone administration were as follows: (1) $40 \mathrm{mg} /$ day for 5 days, 13 patients; (2) $40 \mathrm{mg} /$ day for 3 days, 17 patients; (3) $20 \mathrm{mg} /$ day for 5 days, 9 patients; (4) $20 \mathrm{mg} /$ day for 3 days, 11 patients. Corticosteroid were initiated within a median of 2 days (IQR 1-5 days) of hospital admission. 
Table 2 Variables associated with primary outcomes

\begin{tabular}{|c|c|c|c|c|}
\hline & \multicolumn{2}{|l|}{ Univariate analysis } & \multicolumn{2}{|l|}{ Multivariate analysis } \\
\hline & OR (95\% CI) & $p$ value & $\overline{\text { OR }(95 \% \mathrm{CI})}$ & $p$ value \\
\hline Age (years) & $1.087(1.027-1.152)$ & 0.004 & $1.077(1.011-1.148)$ & 0.022 \\
\hline Male & $2.560(1.115-6.813)$ & 0.035 & $1.054(0.357-4.226)$ & 0.776 \\
\hline Comorbidities & $2.429(1.160-3.941)$ & $<0.001$ & $1.135(1.041-1.848)$ & 0.045 \\
\hline Temperature $\left(>37.3^{\circ} \mathrm{C}\right)$ & $1.836(0.197-3.543)$ & 0.808 & & \\
\hline Respiratory rates $(/ \mathrm{min})$ & $1.051(0.988-1.119)$ & 0.113 & & \\
\hline Heart rates $(/ \mathrm{min})$ & $1.008(0.929-1.034)$ & 0.457 & & \\
\hline $\mathrm{SBP}(\mathrm{mmHg})$ & $1.172(0.983-1.828)$ & 0.748 & & \\
\hline Oxygen saturation (\%) & $0.998(0.739-2.055)$ & 0.424 & & \\
\hline $\mathrm{FiO} 2(\%)$ & $1.128(0.989-1.374)$ & 0.068 & & \\
\hline D-dimer $(\mathrm{ng} / \mathrm{mL})$ & $1.166(1.093-1.271)$ & 0.018 & $1.018(0.974-1.146)$ & 0.128 \\
\hline WBC $\left(10^{9} / \mathrm{L}\right)$ & $1.177(1.037-1.342)$ & 0.025 & $1.143(0.934-1.231)$ & 0.327 \\
\hline Lymphocyte $\left(10^{9} / \mathrm{L}\right)$ & $0.437(0.024-0.940)$ & $<0.001$ & $0.738(0.622-0.877)$ & 0.029 \\
\hline $\mathrm{CRP}(\mathrm{mg} / \mathrm{L})$ & $1.061(1.019-1.140)$ & 0.022 & $1.031(0.985-1.097)$ & 0.185 \\
\hline $\mathrm{LDH}(\mathrm{U} / \mathrm{L})$ & $1.015(1.010-1.022)$ & $<0.001$ & $1.012(1.008-1.017)$ & 0.012 \\
\hline Thymosin- $\alpha$ & $1.208(0.285-5.119)$ & 0.798 & & \\
\hline Hydroxychloroquine & $6.453(0.788-52.859)$ & 0.082 & & \\
\hline Arbidol & $5.447(0.637-42.784)$ & 0.681 & & \\
\hline Lopinavir/ritonavir & $4.377(0.525-38.563)$ & 0.443 & & \\
\hline
\end{tabular}

Primary outcomes included the rate of patients who developed severe disease and mortality. Multivariate analysis was fitted by including the factors associated with primary outcomes in the univariable analyses $(p<0.05)$

$S B P$ systemic blood pressure, FiO2 fraction of inspired $\mathrm{O}_{2}, W B C$ white blood cell, $C R P$ C-reactive protein, $L D H$ lactate dehydrogenase

\section{Variables Associated with Primary Outcomes}

Results of univariate and multivariate logistic regression analyses of primary outcomes are shown in Table 2. Univariate analysis showed that age, male, comorbidities, D-dimer, WBC, lymphocyte, CRP, and LDH were associated with primary outcomes (all $p<0.05$ ). Multivariable analysis identified age (OR 1.077, 95\% CI 1.011-1.148, $p=0.022$ ), comorbidities (OR 1.135, 95\% CI 1.041-1.848, $p=0.045$ ), lymphocyte (OR 0.738, 95\% CI 0.622-0.877, $p=0.029)$, and LDH (OR 1.012, 95\% CI 1.008-1.017, $p=0.012$ ) as the independent variables associated with primary outcomes. Therefore, age, comorbidities, lymphocyte, and LDH were adjusted in baseline characteristics of patients between the corticosteroids group and non-corticosteroids group using PSM.

\section{Baseline Characteristics of Patients After PSM}

The baseline characteristics of patients after PSM are shown in Table 3. After PSM, the 
Table 3 Baseline characteristics of patients after propensity score matching

\begin{tabular}{llll}
\hline & Corticosteroids group & Non-corticosteroids group & $\boldsymbol{p}$ value \\
\hline Number of patients & 55 & 55 & \\
Propensity score & $0.11(0.10-0.14)$ & $0.11(0.10-0.14)$ & 0.869 \\
Age (years) & $59(46-68)$ & $58(43-70)$ & 0.814 \\
Vital signs & & & \\
Temperature $\left({ }^{\circ} \mathrm{C}\right)$ & $37.7(37.2-38.2)$ & $37.5(37.1-38.0)$ & 0.245 \\
Respiratory rates $(/ \mathrm{min})$ & $25(22-27)$ & $25(22-27)$ & 0.314 \\
Heart rates $(/$ min) & $88(79-94)$ & $86(76-95)$ & 0.582 \\
SBP $($ mmHg) & $125(110-142)$ & $122(108-140)$ & 0.671 \\
Oxygen saturation $(\%)$ & $95(94-97)$ & $95(94-97)$ & 0.776 \\
FiO2 $(\%)$ & $41(33-41)$ & $41(33-41)$ & 0.172 \\
Comorbidities, $n(\%)$ & $23(41.8 \%)$ & $25(45.5 \%)$ & 0.701 \\
Hypertension & $19(34.5 \%)$ & $20(36.4 \%)$ & 0.842 \\
Diabetes & $6(10.9 \%)$ & $7(12.7 \%)$ & 0.768 \\
Chronic heart disease & $3(5.5 \%)$ & $2(3.6 \%)$ & 0.647 \\
Chronic liver disease & $1(1.8 \%)$ & 0 & 0.315 \\
Chronic pulmonary disease & $1(1.8 \%)$ & 0 & 0.315 \\
Laboratory parameters at admission & & $0.9(0.8-1.1)$ & 0.415 \\
Lymphocyte $\left(10^{9} / \mathrm{L}\right)$ & $0.9(0.7-1.1)$ & $289(217-351)$ & 0.753 \\
Lactate dehydrogenase $(\mathrm{U} / \mathrm{L})$ & $285(215-357)$ & &
\end{tabular}

$p$ values indicate differences between the corticosteroids group and non-corticosteroids group SBP systemic blood pressure, $\mathrm{FiO} 2$ fraction of inspired $\mathrm{O}_{2}$

baseline characteristics of patients were well balanced between the corticosteroids group and non-corticosteroids group. Thus, the four covariates (age, comorbidities, lymphocyte, and $\mathrm{LDH}$ ) could be considered as being well balanced after adjustment on the propensity score.

\section{Evaluation of Efficacy for Corticosteroids After PSM}

Results of the evaluation of efficacy for corticosteroids after PSM are shown in Table 4. Compared to the non-corticosteroids group, there was a prolonged duration of fever (median 5 vs 3 days, $p<0.001$ ), virus clearance time (median 18 vs 11 days, $p<0.001$ ), and length of hospital stay (median 23 vs 15 days, $p<0.001$ ) in the corticosteroids group. The percentages of antibiotics therapy $(89.1 \%$ vs $23.6 \%, p<0.001)$, use of at least two antibiotics (38.2\% vs $12.7 \%$, $p=0.002)$, and antifungal therapy $(7.3 \%$ vs 0 , $p=0.042)$ were higher in the corticosteroids group than those in the non-corticosteroids group. Meanwhile, compared to the non-corticosteroids group, more patients developed severe disease $(12.7 \%$ vs $1.8 \%, p=0.028)$ in the corticosteroids group. There was no significant difference between the two groups in mortality $(1.8 \%$ vs $0, p=0.315)$. 
Table 4 Evaluation of efficacy for corticosteroids after propensity score matching

\begin{tabular}{llll}
\hline & Corticosteroids & Non-corticosteroids & $p$ value \\
\hline Number of patients & 55 & 55 & \\
Primary outcomes & $7(12.7 \%)$ & $1(1.8 \%)$ & 0.028 \\
Developed severe disease & $1(1.8 \%)$ & 0 & $<.315$ \\
Died & & & $<0.001$ \\
Secondary outcomes & $5(4-7)$ & $3(1-5)$ & $<0.001$ \\
Duration of fever (days) & $18(13-21)$ & $11(8-16)$ & $<0.001$ \\
Virus clearance time (days) & $23(17-28)$ & $13(23.6 \%)$ & $<0.001$ \\
Length of hospital stay (days) & $49(89.1 \%)$ & $7(12.7 \%)$ & 0.002 \\
Antibiotics therapy, $n(\%)$ & $21(38.2 \%)$ & 0 & 0.042 \\
Use of $\geq 2$ antibiotics, $n(\%)$ & $4(7.3 \%)$ & & \\
Antifungal therapy, $n(\%)$ & & & \\
\hline
\end{tabular}

$p$ values indicate differences between the corticosteroids group and non-corticosteroids group
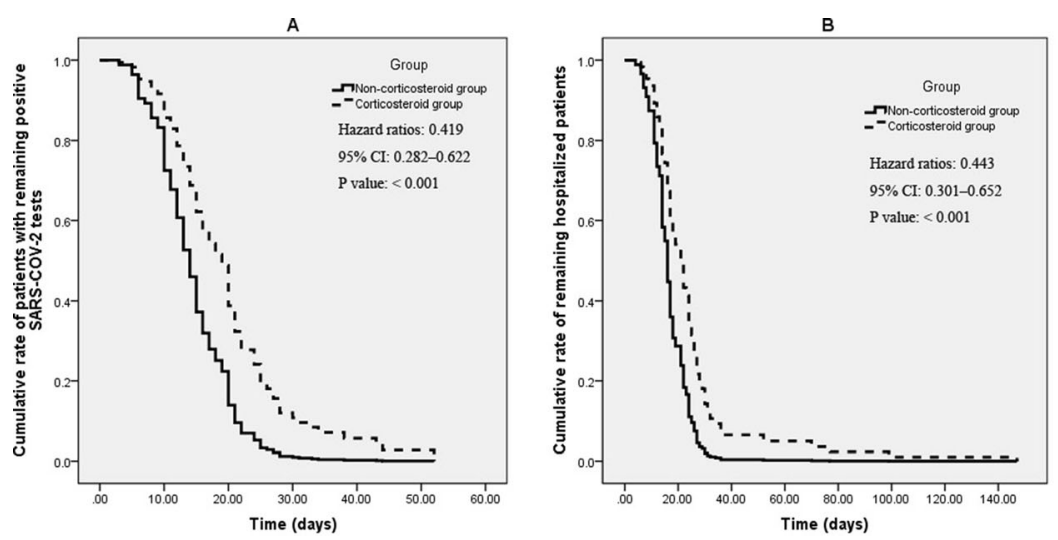

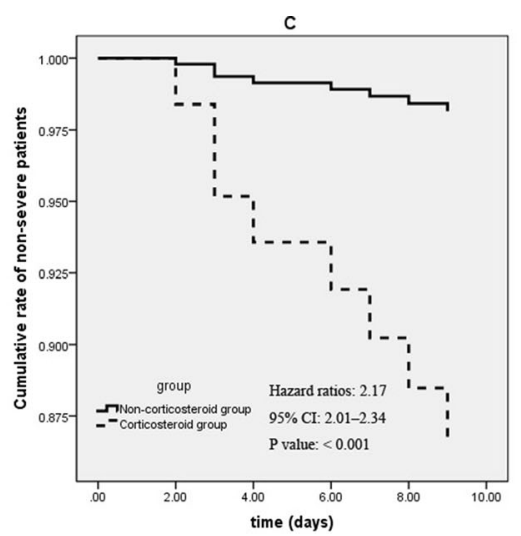

Fig. 2 Cox analysis for comparison of time variables between groups. Cox regression analysis showed significant differences in the virus clearance time (HR 0.419, 95\% CI $0.282-0.622, p<0.001$ ) (a), length of hospital stay (HR $0.443,95 \%$ CI $0.301-0.652, p<0.001)(\mathbf{b})$, and the rate

\section{Cox Analysis for Comparison of Time Variables Between Groups}

Cox regression analysis showed significant differences in the virus clearance time (HR 0.419, 95\% CI 0.282-0.622, $p<0.001$ ) (Fig. 2a), length of hospital stay (HR 0.443, 95\% CI of COVID-19 progression to severe disease (HR 2.17, $95 \%$ CI $2.01-2.34, p<0.001$ ) (c) between the corticosteroids group and non-corticosteroids group. There was a significant rise of $10.9 \%$ in the severe cases among the recipients of corticosteroids vs non-corticosteroids

$0.301-0.652, p<0.001$ ) (Fig. 2b), and the rate of COVID-19 progression to severe disease (HR 2.17, 95\% CI 2.01-2.34, $p<0.001$ ) (Fig. 2c) between the corticosteroids group and noncorticosteroids group. There was a significant rise of $10.9 \%$ in the severe cases among the recipients of corticosteroids vs non- 
corticosteroids. In this study, only one death was observed in the corticosteroids group, and no deaths were observed in the non-corticosteroids group. Therefore, a survivorship curve was unnecessary in this study.

\section{DISCUSSION}

Corticosteroids therapy of patients with COVID-19 is common in clinical practice, but the efficacy remains a major controversy. Some patients with COVID-19 exhibit biphasic disease evolution with a mild presentation followed by a secondary respiratory deterioration. Theoretically, early corticosteroids therapy might reduce inflammatory response, and prevented the progression of COVID-19. However, many questions regarding the use of corticosteroids in the treatment of COVID-19 remain unanswered, including the efficacy, the appropriate timing of initiation, and the dose. In this study, we evaluated the efficacy of early, lowdose, and short-term corticosteroids therapy in adults hospitalized with non-severe COVID-19 pneumonia. Our results indicated that early, low-dose, and short-term corticosteroids therapy was associated with worse clinical outcomes in the treatment of patients with non-severe COVID-19 pneumonia.

Generally, corticosteroids are considered to induce a decrease in body temperature and help alleviate the poisoning symptoms of virus infection including fever, headache, fatigue, myalgia, and so on. However, in this study, compared to the non-corticosteroids group, there was a prolonged duration of fever (median 5 vs 3 days, $p<0.001)$ in the corticosteroids group. Zha et al. [15] also found that there was a prolonged duration of symptoms (median 8 vs 6.5 days) in the corticosteroids group compared to the non-corticosteroids group. Yuan et al. [16] found that there were no statistically significant differences in duration of fever $(9.5 \mathrm{vs}$ 10.2 days, $p=0.28$ ) between patients who received and those who did not receive corticosteroids treatment. On the basis of these studies, we suggested that corticosteroids may not be helpful in shortening the duration of fever. Although the temperature decreased temporarily, it increased again when corticosteroids wear off. The prolonged duration of fever could be attributed to delayed SARS-CoV-2 clearance due to the immunosuppressive effect of corticosteroids.

In this study, compared to the non-corticosteroids group, there was a prolonged virus clearance time (median 18 vs 11 days, $p<0.001)$ in the corticosteroids group. The results were consistent with previous studies. Ling et al. [17] reported that the duration of SARS-CoV-2 RNA in the corticosteroids group was longer than that in the non-corticosteroids group (15 vs 8.0 days, $p=0.013$ ). Ma et al. [18] proposed that SARS-CoV-2 RNA clearance would be delayed because of the immunosuppressive effect of higher dose of glucocorticoids. Li et al. [19] included 10 cohort studies and one randomized clinical trial involving 5249 subjects, and found that corticosteroids use in subjects with SARS-CoV-2, SARS-CoV, and Middle East respiratory syndrome (MERS)-CoV infections delayed virus clearing. A retrospective study by Yuan et al. [16] also found that compared to the non-corticosteroids group, corticosteroids group had longer duration of SARS-CoV-2 shedding (20.3 vs 19.4 days, $p=0.669$ ).

In this study, more patients in the corticosteroids group developed severe disease $(12.7 \%$ vs $1.8 \%, p=0.028$ ) than did in the non-corticosteroids group. The results were consistent with some previous studies. Xu et al., reported a patient with COVID-19 treated with methylprednisolone since day 8 of the disease course. However, his situation worsened and developed respiratory failure and died on day 14 [4]. A meta-analysis included 5270 patients from 15 studies, and found that corticosteroids treatment was associated with higher mortality $(\mathrm{RR}=2.11, \quad 95 \% \quad \mathrm{CI}=1.13-3.94, \quad p=0.019)$, longer length of stay (weighted mean difference $[\mathrm{WMD}]=6.31,95 \% \mathrm{CI}=5.26-7.37, p<0.001)$, and a higher rate of bacterial infection $(\mathrm{RR}=$ $2.08,95 \% \quad \mathrm{CI}=1.54-2.81, \quad p<0.001) \quad$ in patients with COVID-19 [20]. Yuan et al. [16] also found that compared to the non-corticosteroids group, the corticosteroids group had more patients with non-severe COVID-19 pneumonia who developed severe disease 
(11.4\% vs $2.9 \%, p=0.353)$. According to the aforementioned studies, corticosteroids might have a negative effect on lung injury recovery in non-severe COVID-19 pneumonia.

Corticosteroids therapy of severe patients with COVID-19 has been widely studied. Goursaud et al. [21] reported that some selected patients with severe COVID-19 would benefit from wise and timely use of corticosteroids. Yang and Lipes [22] described a case series of 15 patients with COVID-19 admitted to ICU who received corticosteroids in the context of cytokine release syndrome, and found a significant clinical and biochemical association between corticosteroids therapy and improved surrogate outcomes. A meta-analysis found that corticosteroids therapy may reduce mortality for patients with COVID-19 and ARDS (RR 0.72, 95\% CI 0.55-0.93, mean difference $17.3 \%$ lower) [23]. Another meta-analysis also found that methylprednisolone could lower the mortality rate in more severe forms of this condition, such as in ARDS [24].

The RECOVERY Collaborative Group performed a controlled, open-label trial comparing a range of possible treatments in patients who were hospitalized with COVID-19, and randomly assigned patients to receive dexamethasone orally or intravenously (at a dose of $6 \mathrm{mg}$ once daily) for up to 10 days or to receive usual care alone [25]. In the dexamethasone group, the incidence of death was lower than that in the usual care group among patients receiving invasive mechanical ventilation $(29.3 \%$ vs $41.4 \%$, RR $0.64,95 \%$ CI $0.51-0.81$ ) and among those receiving oxygen without invasive mechanical ventilation $(23.3 \%$ vs $26.2 \%$, RR 0.82 , 95\% CI 0.72-0.94) but not among those who were receiving no respiratory support at randomization $(17.8 \%$ vs $14.0 \%$, RR $1.19,95 \%$ CI 0.91-1.55) [25]. In this study, our results indicate that the use of corticosteroids increased the risk of COVID-19 progression to severe disease, increased the use of antibiotics, and prolonged duration of fever, virus clearance time, and length of hospital stay in non-severe COVID-19 pneumonia. Our results were not contradictory with the results of the RECOVERY study, which showed that the use of dexamethasone resulted in lower 28-day mortality among patients with COVID-19 who were receiving either invasive mechanical ventilation or oxygen alone (severe cases mainly) but not among those receiving no respiratory support (non-severe cases mainly).

This study has several limitations. First, the study is retrospective. The uses of corticosteroids were decided by joint discussion of five experts from the Shanghai Medical Expert Group for the Treatment of COVID-19, on the basis of the demographics, laboratory parameters, and CT scans of patients. As a tentative therapy, considering the side effect and dubious curative effect, corticosteroids were used selectively for patients with more risk factors for a worse evolution of COVID-19 (or even more severe) than control patients. In order to reduce the biases and weaknesses caused by selective deviation, propensity score was used to balance the differences between the corticosteroids group and control group. However, propensity score is limited by adjusting for observed variables only; it cannot account for residual confounding of many of variables. Therefore, our results need confirmation in a prospective randomized clinical trial. Second, the dose and duration are critical to evaluate the efficacy of corticosteroids. In this study, the actual dose (20 mg/day or $40 \mathrm{mg} /$ day) and duration (3 days or 5 days) of corticosteroids are absolute rather than calculation by milligrams per kilo. The dose and duration of corticosteroids were not randomized, but decided by joint discussions of five experts from the Shanghai Medical Expert Group for the Treatment of COVID-19. The selective biases in dose and duration of corticosteroids might affect the efficacy of corticosteroids in non-severe COVID-19 pneumonia. Third, no children were enrolled in this study because children with COVID-19 were hospitalized at another designated hospital in Shanghai.

\section{CONCLUSION}

Our data indicate that the use of corticosteroids increased the risk of COVID-19 progression to severe disease, increased the use of antibiotics, and prolonged duration of fever, virus clearance 
time, and length of hospital stay in patients with non-severe COVID-19 pneumonia. In hospitalized adult patients with non-severe COVID-19 pneumonia, early, low-dose, and short-term corticosteroids were associated with worse clinical outcomes. Our results did not support the use of corticosteroids in patients with non-severe COVID-19 pneumonia.

\section{ACKNOWLEDGEMENTS}

We thank the doctors (Shui-Bao Xu, Yi-Xiao Lin, Feng Li, Tao Li, Zhiping Qian, Jun Chen, BiJie $\mathrm{Hu}$, Sheng Wang, En-Qiang Mao, Lei Zhu, Wen-Hong Zhang, Yinzhong Shen) for their efforts in the diagnosis and treatment of patients.

Funding. This study was supported by grant no. 17411969700 from Shanghai Association for Science and Technology and grant no. 19YF1441200 from Shanghai Sailing Plan Program. The Rapid Service Fees were funded by the authors. The funding organizations are public institutions and had no role in the design and conduct of the study; collection, management, and analysis of the data; or preparation, review, and approval of the manuscript.

Authorship. All named authors meet the International Committee of Medical Journal Editors (ICMJE) criteria for authorship for this article, take responsibility for the integrity of the work as a whole, and have given their approval for this version to be published.

Authorship Contributions. Study concept and design: Liang Chen. Data collection: Qiang $\mathrm{Li}$, Weixia Li, Yinpeng Jin, Wei Xu, Chenlu Huang, and Li Li. Analysis and interpretation of data: Yuxian Huang, Qingchun Fu, and Liang Chen. Drafting of the manuscript: Qiang Li, Weixia Li, Yinpeng Jin, Wei $\mathrm{Xu}$, and Chenlu Huang. Critical revision of the manuscript: Liang Chen.

Disclosures. Qiang Li, Weixia Li, Yinpeng Jin, Wei $\mathrm{Xu}$, Chenlu Huang, Li Li, Yuxian
Huang, Qingchun Fu and Liang Chen have nothing to declare.

Compliance with Ethics Guidelines. The ethics committee of Shanghai Public Health Clinical Center approved the study protocol including any relevant details (YJ-2020-S09501). Although this is a retrospective study, at admission, all patients provided verbal consent that their clinical data might be used for medical study. When we performed the study, all personal information of patients was de-identified to protect privacy. This study was performed in accordance with the declaration of Helsinki.

Data Availability. We declare that materials described in the manuscript, including all relevant raw data, will be freely available to any scientist wishing to use them for non-commercial purposes, without breaching participant confidentiality. The supporting data can be accessed from the corresponding author upon request.

Open Access. This article is licensed under a Creative Commons Attribution-NonCommercial 4.0 International License, which permits any non-commercial use, sharing, adaptation, distribution and reproduction in any medium or format, as long as you give appropriate credit to the original author(s) and the source, provide a link to the Creative Commons licence, and indicate if changes were made. The images or other third party material in this article are included in the article's Creative Commons licence, unless indicated otherwise in a credit line to the material. If material is not included in the article's Creative Commons licence and your intended use is not permitted by statutory regulation or exceeds the permitted use, you will need to obtain permission directly from the copyright holder. To view a copy of this licence, visit http://creativecommons.org/licenses/by$\mathrm{nc} / 4.0 /$. 


\section{REFERENCES}

1. Huang C, Wang Y, Li X, et al. Clinical features of patients infected with 2019 novel coronavirus in Wuhan, China. Lancet. 2020;395:497-506.

2. World Health Organization. Coronavirus disease situation reports-166. 2019. https://www.who.int/ docs/default-source/coronaviruse/situation-reports/ 20200704-covid-19-sitrep-166. Accessed 4 Jul 2020.

3. Wang D, Hu B, Hu C, et al. Clinical characteristics of 138 hospitalized patients with 2019 novel coronavirus-infected pneumonia in Wuhan, China. JAMA. 2020;323:1061-9.

4. Xu Z, Shi L, Wang Y, et al. Pathological findings of COVID-19 associated with acute respiratory distress syndrome. Lancet Respir Med. 2020;8:P420-2. https://doi.org/10.1016/S2213-2600(20)30076-X.

5. Chen N, Zhou M, Dong X, et al. Epidemiological and clinical characteristics of 99 cases of 2019 novel coronavirus pneumonia in Wuhan, China: a descriptive study. Lancet. 2020;395:507-13.

6. Chen RC, Tang XP, Tan SY, et al. Treatment of severe acute respiratory syndrome with glucosteroids: the Guangzhou experience. Chest. 2006;129:1441-52.

7. Russell B, Moss C, Rigg A, et al. COVID-19 and treatment with NSAIDs and corticosteroids: should we be limiting their use in the clinical setting? Ecancermedicalscience. 2020;14:1023.

8. Han Y, Jiang M, Xia D, et al. COVID-19 in a patient with long-term use of glucocorticoids: a study of a familial cluster. Clin Immunol. 2020;214:108413.

9. Tang C, Wang Y, Lv H, et al. Caution against corticosteroid-based COVID-19 treatment. Lancet. 2020;395:1759-60.

10. WHO. Clinical management of severe acute respiratory infection when Novel coronavirus (nCoV) infection is suspected: interim guidance. 2020. https://www.who.int/internal-publicationsdetail/ clinical-management-of-severe-acute-respiratoryinfection-when-novel-coronavirus-(ncov)infection-is-suspected. Accessed 5 Feb 2020.

11. National Health Commision of the People's Republic of China. New coronavirus pneumonia prevention and control program (the fifth edition). 2020 . http://www.nhc.gov.cn/yzygj/s7653p/ 202002/3b09b894ac9b4204a79db5b8912d4440. shtml. Accessed 5 Feb 2020.

12. Haukoos JS, Lewis RJ. The propensity score. JAMA. 2015;314:1637-8.
13. Kim SH, Hong SB, Yun SC, et al. Corticosteroid treatment in critically ill patients with pandemic influenza A/H1N1 2009 infection: analytic strategy using propensity scores. Am J Respir Crit Care Med. 2011;183:1207-14.

14. Benedetto U, Head SJ, Angelini GD, et al. Statistical primer: propensity score matching and its alternatives. Eur J Cardiothorac Surg. 2018;53:1112-7.

15. Zha L, Li S, Pan L, et al. Corticosteroid treatment of patients with coronavirus disease 2019 (COVID-19). Med J Aust. 2020;212:416-20.

16. Yuan $\mathrm{M}, \mathrm{Xu} \mathrm{X}, \mathrm{Xia} \mathrm{D}$, et al. Effects of corticosteroid treatment for non-severe COVID-19 pneumonia: a propensity score-based analysis. Shock. 2020. https://doi.org/10.1097/SHK.0000000000001574.

17. Ling $\mathrm{Y}, \mathrm{Xu} \mathrm{S} \mathrm{B}$, Lin $\mathrm{Y} \mathrm{X}$, et al. Persistence and clearance of viral RNA in 2019 novel coronavirus disease rehabilitation patients. Chin Med J (Engl). 2020;133:1039-43.

18. Ma S Q, Zhang J, Wang Y S, et al. Glucocorticoid therapy delays the clearance of SARS-CoV-2 RNA in an asymptomatic COVID-19 patient. J Med Virol. 2020. https://doi.org/10.1002/jmv.26086.

19. Li H, Chen C, Hu F, et al. Impact of corticosteroid therapy on outcomes of persons with SARS-CoV-2, SARS-CoV, or MERS-CoV infection: a systematic review and meta-analysis. Leukemia. 2020;34: 1503-11.

20. Yang Z, Liu J, Zhou Y, et al. The effect of corticosteroid treatment on patients with coronavirus infection: a systematic review and meta-analysis. J Infect. 2020;81:e13-20.

21. Goursaud S, Descamps R, Daubin C, et al. Corticosteroid use in selected patients with severe acute respiratory distress syndrome related to COVID-19. J Infect. 2020;81:e89-e90.

22. Yang S S, Lipes J. Corticosteroids for critically ill COVID-19 patients with cytokine release syndrome: a limited case series. Can J Anaesth. 2020. https://doi.org/10.1007/s12630-020-01700-w.

23. Ye Z, Wang Y, Colunga-Lozano LE, et al. Efficacy and safety of corticosteroids in COVID-19 based on evidence for COVID-19, other coronavirus infections, influenza, community-acquired pneumonia and acute respiratory distress syndrome: a systematic review and meta-analysis. CMAJ. 2020;192(27): E756-67.

24. Veronese N, Demurtas J, Yang L, et al. Use of corticosteroids in coronavirus disease 2019 pneumonia: a systematic review of the literature. Front Med. 2020;7:170. 
25. Horby P, Lim WS, Emberson JR, et al. Dexamethasone in hospitalized patients with Covid-19- preliminary report. N Engl J Med. 2020. https://doi. org/10.1056/NEJMoa2021436. 